\title{
A new solution to the Rhoades' open problem with an application
}

\author{
Nihal Özgür \\ Balıkesir University, \\ Department of Mathematics, \\ 10145 Balıkesir, TURKEY \\ email: nihal@balikesir.edu.tr
}

\author{
Nihal Taş \\ Balıkesir University, \\ Department of Mathematics, \\ 10145 Balıkesir, TURKEY \\ email: nihaltas@balikesir .edu.tr
}

\begin{abstract}
We give a new solution to the Rhoades' open problem on the discontinuity at fixed point via the notion of an S-metric. To do this, we develop a new technique by means of the notion of a Zamfirescu mapping. Also, we consider a recent problem called the "fixed-circle problem" and propose a new solution to this problem as an application of our technique.
\end{abstract}

\section{Introduction and preliminaries}

Fixed-point theory has been extensively studied by various aspects. One of these is the discontinuity problem at fixed points (see $[1,2,3,4,5,6,24,25,26$, 27] for some examples). Discontinuous functions have been widely appeared in many areas of science such as neural networks (for example, see [7, 12, 13, 14]). In this paper, we give a new solution to the Rhoades' open problem (see [28] for more details) on the discontinuity at fixed point in the setting of an Smetric space which is a recently introduced generalization of a metric space. S-metric spaces were introduced in [29] by Sedgi et al., as follows:

Definition 1 [29] Let $\mathrm{X}$ be a nonempty set and $\mathcal{S}: \mathrm{X} \times \mathrm{X} \times \mathrm{X} \rightarrow[0, \infty)$ a function satisfying the following conditions for all $x, y, z, a \in X$ :

2010 Mathematics Subject Classification: Primary 54H25; Secondary 47H09, 47H10 Key words and phrases: discontinuity, fixed point, S-metric, fixed-circle problem 
S1) $\mathcal{S}(x, y, z)=0$ if and only if $x=y=z$,

S2) $\mathcal{S}(x, y, z) \leq \mathcal{S}(x, x, a)+\mathcal{S}(y, y, a)+\mathcal{S}(z, z, a)$.

Then $\mathcal{S}$ is called an $\mathrm{S}$-metric on $\mathrm{X}$ and the pair $(\mathrm{X}, \mathcal{S})$ is called an $\mathrm{S}$-metric space.

Relationships between a metric and an S-metric were given as follows:

Lemma 1 [9] Let $(\mathrm{X}, \mathrm{d})$ be a metric space. Then the following properties are satisfied:

1. $\mathcal{S}_{\mathrm{d}}(x, y, z)=\mathrm{d}(x, z)+\mathrm{d}(\mathrm{y}, z)$ for all $\mathrm{x}, \mathrm{y}, z \in \mathrm{X}$ is an S-metric on $\mathrm{X}$.

2. $x_{n} \rightarrow x$ in $(X, d)$ if and only if $x_{n} \rightarrow x$ in $\left(X, \mathcal{S}_{\mathrm{d}}\right)$.

3. $\left\{x_{n}\right\}$ is Cauchy in $(\mathrm{X}, \mathrm{d})$ if and only if $\left\{x_{\mathrm{n}}\right\}$ is Cauchy in $\left(\mathrm{X}, \mathcal{S}_{\mathrm{d}}\right)$.

4. $(\mathrm{X}, \mathrm{d})$ is complete if and only if $\left(\mathrm{X}, \mathcal{S}_{\mathrm{d}}\right)$ is complete.

The metric $\mathcal{S}_{\mathrm{d}}$ was called as the $\mathrm{S}$-metric generated by d [17]. Some examples of an $S$-metric which is not generated by any metric are known (see $[9,17]$ for more details).

Furthermore, Gupta claimed that every S-metric on $X$ defines a metric $d_{S}$ on $\mathrm{X}$ as follows:

$$
\mathrm{d}_{\mathrm{S}}(\mathrm{x}, \mathrm{y})=\mathcal{S}(\mathrm{x}, \mathrm{x}, \mathrm{y})+\mathcal{S}(\mathrm{y}, \mathrm{y}, \mathrm{x}),
$$

for all $x, y \in X[8]$. However, since the triangle inequality does not satisfied for all elements of $X$ everywhen, the function $d_{S}(x, y)$ defined in (1) does not always define a metric (see [17]).

In the following, we see an example of an S-metric which is not generated by any metric.

Example 1 [17] Let $\mathrm{X}=\mathbb{R}$ and the function $\mathcal{S}: \mathrm{X} \times \mathrm{X} \times \mathrm{X} \rightarrow[0, \infty)$ be defined as

$$
\mathcal{S}(x, y, z)=|x-z|+|x+z-2 y|,
$$

for all $x, y, z \in \mathbb{R}$. Then $\mathcal{S}$ is an $S$-metric which is not generated by any metric and the pair $(\mathrm{X}, \mathcal{S})$ is an $\mathrm{S}$-metric space.

The following lemma will be used in the next sections.

Lemma $2[29]$ Let $(\mathrm{X}, \mathcal{S})$ be an $\mathrm{S}$-metric space. Then we have

$$
\mathcal{S}(x, x, y)=\mathcal{S}(y, y, x) .
$$


In this paper, our aim is to obtain a new solution to the Rhoades' open problem on the existence of a contractive condition which is strong enough to generate a fixed point but which does not force the map to be continuous at the fixed point. To do this, we inspire of a result of Zamfirescu given in [33].

On the other hand, a recent aspect to the fixed point theory is to consider geometric properties of the set Fix( $\mathrm{T})$, the fixed point set of the self-mapping T. Fixed-circle problem (resp. fixed-disc problem) have been studied in this context (see $[6,18,19,20,21,22,23,26,27,30,31]$ ). As an application, we present a new solution to these problems. We give necessary examples to support our theoretical results.

\section{Main results}

From now on, we assume that $(X, \mathcal{S})$ is an $S$-metric space and $\mathrm{T}: \mathrm{X} \rightarrow \mathrm{X}$ is a self-mapping. In this section, we use the numbers defined as

$$
M_{z}(x, y)=\max \left\{a d(x, y), \frac{b}{2}[d(x, T x)+d(y, T y)], \frac{c}{2}[d(x, T y)+d(y, T x)]\right\}
$$

and

$$
M_{z}^{S}(x, y)=\max \left\{\begin{array}{c}
a \mathcal{S}(x, x, y), \frac{b}{2}[\mathcal{S}(x, x, T x)+\mathcal{S}(y, y, T y)] \\
\frac{c}{2}[\mathcal{S}(x, x, T y)+\mathcal{S}(y, y, T x)]
\end{array}\right\}
$$

where $a, b \in[0,1)$ and $c \in\left[0, \frac{1}{2}\right]$.

We give the following theorem as a new solution to the Rhoades' open problem.

Theorem 1 Let $(\mathrm{X}, \mathcal{S})$ be a complete $\mathrm{S}$-metric space and $\mathrm{T}$ a self-mapping on $\mathrm{X}$ satisfying the conditions

i) There exists a function $\phi: \mathbb{R}^{+} \rightarrow \mathbb{R}^{+}$such that $\phi(\mathrm{t})<\mathrm{t}$ for each $\mathrm{t}>0$ and

$$
\mathcal{S}(\mathrm{T} x, \mathrm{~T} x, \mathrm{~T} y) \leq \phi\left(M_{z}^{S}(x, y)\right)
$$

for all $\mathrm{x}, \mathrm{y} \in \mathrm{X}$,

ii) There exists a $\delta=\delta(\varepsilon)>0$ such that $\varepsilon<M_{z}^{S}(x, y)<\varepsilon+\delta$ implies $\mathcal{S}(\mathrm{Tx}, \mathrm{Tx}, \mathrm{Ty}) \leq \varepsilon$ for a given $\varepsilon>0$.

Then $\mathrm{T}$ has a unique fixed point $\mathrm{u} \in \mathrm{X}$. Also, $\mathrm{T}$ is discontinuous at $\mathrm{u}$ if and only if $\lim _{x \rightarrow \mathfrak{u}} M_{z}^{S}(x, u) \neq 0$. 
Proof. At first, we define the number

$$
\xi=\max \left\{a, \frac{2}{2-b}, \frac{c}{2-2 c}\right\} .
$$

Clearly, we have $\xi<1$.

By the condition (i), there exists a function $\phi: \mathbb{R}^{+} \rightarrow \mathbb{R}^{+}$such that $\phi(t)<t$ for each $t>0$ and

$$
\mathcal{S}(\mathrm{T} x, \mathrm{~T} x, \mathrm{~T} y) \leq \phi\left(\mathrm{M}_{z}^{\mathrm{S}}(x, y)\right),
$$

for all $x, y \in X$. Using the properties of $\phi$, we obtain

$$
\mathcal{S}(\mathrm{T} x, \mathrm{~T} x, \mathrm{~T} y)<M_{z}^{S}(x, y),
$$

whenever $M_{z}^{S}(x, y)>0$.

Let us consider any $x_{0} \in X$ with $x_{0} \neq T x_{0}$ and define a sequence $\left\{x_{n}\right\}$ as $x_{n+1}=T x_{n}=T^{n} x_{0}$ for all $n=0,1,2,3, \ldots$. Using the condition (i) and the inequality (2), we get

$$
\begin{aligned}
& \mathcal{S}\left(x_{n}, x_{n}, x_{n+1}\right)=\mathcal{S}\left(T x_{n-1}, T x_{n-1}, T x_{n}\right) \leq \phi\left(M_{z}^{S}\left(x_{n-1}, x_{n}\right)\right) \\
& <M_{z}^{S}\left(x_{n-1}, x_{n}\right) \\
& =\max \left\{\begin{array}{c}
a \mathcal{S}\left(x_{n-1}, x_{n-1}, x_{n}\right), \\
\frac{b}{2}\left[\mathcal{S}\left(x_{n-1}, x_{n-1}, T x_{n-1}\right)+\mathcal{S}\left(x_{n}, x_{n}, T x_{n}\right)\right], \\
\frac{c}{2}\left[\mathcal{S}\left(x_{n-1}, x_{n-1}, T x_{n}\right)+\mathcal{S}\left(x_{n}, x_{n}, T x_{n-1}\right)\right]
\end{array}\right\} \\
& =\max \left\{\begin{array}{c}
a \mathcal{S}\left(x_{n-1}, x_{n-1}, x_{n}\right), \\
\frac{b}{2}\left[\mathcal{S}\left(x_{n-1}, x_{n-1}, x_{n}\right)+\mathcal{S}\left(x_{n}, x_{n}, x_{n+1}\right)\right], \\
\frac{c}{2}\left[\mathcal{S}\left(x_{n-1}, x_{n-1}, x_{n+1}\right)+\mathcal{S}\left(x_{n}, x_{n}, x_{n}\right)\right]
\end{array}\right\} \\
& =\max \left\{\begin{array}{c}
a \mathcal{S}\left(x_{n-1}, x_{n-1}, x_{n}\right), \\
\frac{b}{2}\left[\mathcal{S}\left(x_{n-1}, x_{n-1}, x_{n}\right)+\mathcal{S}\left(x_{n}, x_{n}, x_{n+1}\right)\right], \\
\frac{c}{2} \mathcal{S}\left(x_{n-1}, x_{n-1}, x_{n+1}\right)
\end{array}\right\} .
\end{aligned}
$$

Assume that $M_{z}^{S}\left(x_{n-1}, x_{n}\right)=a \mathcal{S}\left(x_{n-1}, x_{n-1}, x_{n}\right)$. Then using the inequality (3), we have

$\mathcal{S}\left(x_{n}, x_{n}, x_{n+1}\right)<a \mathcal{S}\left(x_{n-1}, x_{n-1}, x_{n}\right) \leq \xi \mathcal{S}\left(x_{n-1}, x_{n-1}, x_{n}\right)<\mathcal{S}\left(x_{n-1}, x_{n-1}, x_{n}\right)$ and so

$$
\mathcal{S}\left(x_{n}, x_{n}, x_{n+1}\right)<\mathcal{S}\left(x_{n-1}, x_{n-1}, x_{n}\right) .
$$


Let $M_{z}^{S}\left(x_{n-1}, x_{n}\right)=\frac{b}{2}\left[\mathcal{S}\left(x_{n-1}, x_{n-1}, x_{n}\right)+\mathcal{S}\left(x_{n}, x_{n}, x_{n+1}\right)\right]$. Again using the inequality (3), we get

$$
\mathcal{S}\left(x_{n}, x_{n}, x_{n+1}\right)<\frac{b}{2}\left[\mathcal{S}\left(x_{n-1}, x_{n-1}, x_{n}\right)+\mathcal{S}\left(x_{n}, x_{n}, x_{n+1}\right)\right],
$$

which implies

$$
\left(1-\frac{b}{2}\right) \mathcal{S}\left(x_{n}, x_{n}, x_{n+1}\right)<\frac{b}{2} \mathcal{S}\left(x_{n-1}, x_{n-1}, x_{n}\right)
$$

and hence

$$
\mathcal{S}\left(x_{n}, x_{n}, x_{n+1}\right)<\frac{b}{2-b} \mathcal{S}\left(x_{n-1}, x_{n-1}, x_{n}\right) \leq \xi \mathcal{S}\left(x_{n-1}, x_{n-1}, x_{n}\right) .
$$

This yields

$$
\mathcal{S}\left(x_{n}, x_{n}, x_{n+1}\right)<\mathcal{S}\left(x_{n-1}, x_{n-1}, x_{n}\right) .
$$

Suppose that $M_{z}^{S}\left(x_{n-1}, x_{n}\right)=\frac{c}{2} \mathcal{S}\left(x_{n-1}, x_{n-1}, x_{n+1}\right)$. Then using the inequality (3), Lemma 2 and the condition (S2), we obtain

$$
\begin{aligned}
\mathcal{S}\left(x_{n}, x_{n}, x_{n+1}\right) & <\frac{c}{2} \mathcal{S}\left(x_{n-1}, x_{n-1}, x_{n+1}\right)=\frac{c}{2} \mathcal{S}\left(x_{n+1}, x_{n+1}, x_{n-1}\right) \\
& \leq \frac{c}{2}\left[\mathcal{S}\left(x_{n-1}, x_{n-1}, x_{n}\right)+2 \mathcal{S}\left(x_{n+1}, x_{n+1}, x_{n}\right)\right] \\
& =\frac{c}{2} \mathcal{S}\left(x_{n-1}, x_{n-1}, x_{n}\right)+c \mathcal{S}\left(x_{n+1}, x_{n+1}, x_{n}\right) \\
& =\frac{c}{2} \mathcal{S}\left(x_{n-1}, x_{n-1}, x_{n}\right)+c \mathcal{S}\left(x_{n}, x_{n}, x_{n+1}\right)
\end{aligned}
$$

which implies

$$
(1-c) \mathcal{S}\left(x_{n}, x_{n}, x_{n+1}\right)<\frac{c}{2} \mathcal{S}\left(x_{n-1}, x_{n-1}, x_{n}\right) .
$$

Considering this, we find

$$
\mathcal{S}\left(x_{n}, x_{n}, x_{n+1}\right)<\frac{c}{2(1-c)} \mathcal{S}\left(x_{n-1}, x_{n-1}, x_{n}\right) \leq \xi \mathcal{S}\left(x_{n-1}, x_{n-1}, x_{n}\right)
$$

and so

$$
\mathcal{S}\left(x_{n}, x_{n}, x_{n+1}\right)<\mathcal{S}\left(x_{n-1}, x_{n-1}, x_{n}\right) .
$$

If we set $\alpha_{n}=\mathcal{S}\left(x_{n}, x_{n}, x_{n+1}\right)$, then by the inequalities (4), (5) and (6), we find

$$
\alpha_{n}<\alpha_{n-1},
$$


that is, $\alpha_{n}$ is a strictly decreasing sequence of positive real numbers whence the sequence $\alpha_{n}$ tends to a limit $\alpha \geq 0$.

Assume that $\alpha>0$. There exists a positive integer $k \in \mathbb{N}$ such that $n \geq k$ implies

$$
\alpha<\alpha_{n}<\alpha+\delta(\alpha)
$$

Using the condition (ii) and the inequality (7), we get

$$
\mathcal{S}\left(T x_{n-1}, T x_{n-1}, T x_{n}\right)=\mathcal{S}\left(x_{n}, x_{n}, x_{n+1}\right)=\alpha_{n}<\alpha,
$$

for $n \geq k$. Then the inequality (9) contradicts to the inequality (8). Therefore, it should be $\alpha=0$.

Now we prove that $\left\{x_{n}\right\}$ is a Cauchy sequence. Let us fix an $\varepsilon>0$. Without loss of generality, we suppose that $\delta(\varepsilon)<\varepsilon$. There exists $k \in \mathbb{N}$ such that

$$
\mathcal{S}\left(x_{n}, x_{n}, x_{n+1}\right)=\alpha_{n}<\frac{\delta}{4},
$$

for $n \geq k$ since $\alpha_{n} \rightarrow 0$. Using the mathematical induction and the Jachymski's technique (see $[10,11]$ for more details), we show

$$
\mathcal{S}\left(x_{k}, x_{k}, x_{k+n}\right)<\varepsilon+\frac{\delta}{2},
$$

for any $n \in \mathbb{N}$. At first, the inequality (10) holds for $n=1$ since

$$
\mathcal{S}\left(x_{k}, x_{k}, x_{k+1}\right)=\alpha_{k}<\frac{\delta}{4}<\varepsilon+\frac{\delta}{2} .
$$

Assume that the inequality (10) holds for some $\mathrm{n}$. We show that the inequality (10) holds for $n+1$. By the condition (S2), we get

$$
\mathcal{S}\left(x_{k}, x_{k}, x_{k+n+1}\right) \leq 2 \mathcal{S}\left(x_{k}, x_{k}, x_{k+1}\right)+\mathcal{S}\left(x_{k+n+1}, x_{k+n+1}, x_{k+1}\right) .
$$

From Lemma 2, we have

$$
\mathcal{S}\left(x_{k+n+1}, x_{k+n+1}, x_{k+1}\right)=\mathcal{S}\left(x_{k+1}, x_{k+1}, x_{k+n+1}\right)
$$

and so it suffices to prove

$$
\mathcal{S}\left(x_{k+1}, x_{k+1}, x_{k+n+1}\right) \leq \varepsilon .
$$

To do this, we show

$$
M_{z}^{S}\left(x_{k}, x_{k+n}\right) \leq \varepsilon+\delta .
$$


Then we find

$$
\begin{aligned}
& a \mathcal{S}\left(x_{k}, x_{k}, x_{k+n}\right)<\mathcal{S}\left(x_{k}, x_{k}, x_{k+n}\right)<\varepsilon+\frac{\delta}{2}, \\
& \quad \frac{b}{2}\left[\mathcal{S}\left(x_{k}, x_{k}, x_{k+1}\right)+\mathcal{S}\left(x_{k+n}, x_{k+n}, x_{k+n+1}\right)\right] \\
& <\mathcal{S}\left(x_{k}, x_{k}, x_{k+1}\right)+\mathcal{S}\left(x_{k+n}, x_{k+n}, x_{k+n+1}\right) \\
& <\frac{\delta}{4}+\frac{\delta}{4}=\frac{\delta}{2}
\end{aligned}
$$

and

$$
\begin{aligned}
& \frac{c}{2}\left[\mathcal{S}\left(x_{k}, x_{k}, x_{k+n+1}\right)+\mathcal{S}\left(x_{k+n}, x_{k+n}, x_{k+1}\right)\right] \\
& \leq \frac{c}{2}\left[4 \mathcal{S}\left(x_{k}, x_{k}, x_{k+1}\right)+\mathcal{S}\left(x_{k+1}, x_{k+1}, x_{k+1+n}\right)+\mathcal{S}\left(x_{k}, x_{k}, x_{k+n}\right)\right] \\
& =c\left[2 \mathcal{S}\left(x_{k}, x_{k}, x_{k+1}\right)+\frac{\mathcal{S}\left(x_{k+1}, x_{k+1}, x_{k+1+n}\right)}{2}+\frac{\mathcal{S}\left(x_{k}, x_{k}, x_{k+n}\right)}{2}\right] \\
& <c\left[\frac{\delta}{2}+\varepsilon+\frac{\delta}{2}\right]<\varepsilon+\delta .
\end{aligned}
$$

Using the definition of $M_{z}^{S}\left(x_{k}, x_{k+n}\right)$, the condition (ii) and the inequalities (10) and (11), we obtain

$$
M_{z}^{S}\left(x_{k}, x_{k+n}\right) \leq \varepsilon+\delta
$$

and so

$$
\mathcal{S}\left(x_{\mathrm{k}+1}, x_{\mathrm{k}+1}, \mathrm{x}_{\mathrm{k}+\mathrm{n}+1}\right) \leq \varepsilon .
$$

Hence we get

$$
\mathcal{S}\left(x_{\mathrm{k}}, \mathrm{x}_{\mathrm{k}}, \mathrm{x}_{\mathrm{k}+\mathrm{n}+1}\right)<\varepsilon+\frac{\delta}{2},
$$

whence $\left\{x_{n}\right\}$ is Cauchy. From the completeness hypothesis, there exists a point $u \in X$ such that $x_{n} \rightarrow u$ for $n \rightarrow \infty$. Also we get

$$
\lim _{n \rightarrow \infty} T x_{n}=\lim _{n \rightarrow \infty} x_{n+1}=u .
$$

Now we prove that $u$ is a fixed point of $T$. On the contrary, assume that $u$ is not a fixed point of $\mathrm{T}$. Then using the condition (i) and the property of $\phi$, we obtain

$$
\mathcal{S}\left(T u, T u, T x_{n}\right) \leq \phi\left(M_{z}^{S}\left(u, x_{n}\right)\right)<M_{z}^{S}\left(u, x_{n}\right)
$$




$$
=\max \left\{\begin{array}{c}
a \mathcal{S}\left(u, u, x_{n}\right), \frac{b}{2}\left[\mathcal{S}(u, u, T u)+\mathcal{S}\left(x_{n}, x_{n}, T x_{n}\right)\right], \\
\frac{c}{2}\left[\mathcal{S}\left(u, u, T x_{n}\right)+\mathcal{S}\left(x_{n}, x_{n}, T u\right)\right]
\end{array}\right\} .
$$

Using Lemma 2 and taking limit for $n \rightarrow \infty$, we find

$$
\mathcal{S}(\mathrm{Tu}, \mathrm{Tu}, \mathrm{u})<\max \left\{\frac{\mathrm{b}}{2} \mathcal{S}(\mathrm{u}, \mathrm{u}, \mathrm{Tu}), \frac{\mathrm{c}}{2} \mathcal{S}(\mathrm{u}, \mathrm{u}, \mathrm{Tu})\right\}<\mathcal{S}(\mathrm{Tu}, \mathrm{Tu}, \mathrm{u}),
$$

a contradiction. It should be $T u=u$. We show that $u$ is the unique fixed point of $T$. Let $v$ be another fixed point of $T$ such that $u \neq v$. From the condition (i) and Lemma 2, we have

$$
\left.\begin{array}{rl}
\mathcal{S}(\mathrm{Tu}, \mathrm{Tu}, \mathrm{T} v) & =\mathcal{S}(\mathrm{u}, \mathrm{u}, v) \leq \phi\left(M_{z}^{\mathrm{S}}(\mathrm{u}, v)\right)<M_{z}^{S}(\mathrm{u}, v) \\
& =\max \left\{\begin{array}{c}
a \mathcal{S}(u, u, v), \frac{\mathrm{b}}{2}[\mathcal{S}(\mathrm{u}, \mathrm{u}, \mathrm{Tu})+\mathcal{S}(v, v, \mathrm{~T} v)], \\
\frac{\mathrm{c}}{2}[\mathcal{S}(\mathrm{u}, \mathrm{u}, \mathrm{T} v)+\mathcal{S}(v, v, \mathrm{Tu})]
\end{array}\right\} \\
& =\max \{a \mathcal{S}(u, u, v), c \mathcal{S}(u, u, v)\}<\mathcal{S}(u, u, v),
\end{array}\right\}
$$

a contradiction. So it should be $u=v$. Therefore, $T$ has a unique fixed point $u \in X$.

Finally, we prove that $T$ is discontinuous at $u$ if and only if $\lim _{x \rightarrow \mathfrak{u}} M_{z}^{S}(x, \mathfrak{u}) \neq$ 0 . To do this, we can easily show that $T$ is continuous at $u$ if and only if $\lim _{x \rightarrow u} M_{z}^{S}(x, u)=0$. Suppose that $T$ is continuous at the fixed point $u$ and $x_{n} \rightarrow u$. Hence we get $T x_{n} \rightarrow T u=u$ and using the condition (S2), we find

$$
\mathcal{S}\left(x_{n}, x_{n}, T x_{n}\right) \leq 2 \mathcal{S}\left(x_{n}, x_{n}, u\right)+\mathcal{S}\left(T x_{n}, T x_{n}, u\right) \rightarrow 0,
$$

as $x_{n} \rightarrow u$. So we get $\lim _{x_{n} \rightarrow \mathfrak{u}} M_{z}^{S}\left(x_{n}, \mathfrak{u}\right)=0$. On the other hand, assume $\lim _{x_{n} \rightarrow u} M_{z}^{S}\left(x_{n}, u\right)=0$. Then we obtain $\mathcal{S}\left(x_{n}, x_{n}, T x_{n}\right) \rightarrow 0$ as $x_{n} \rightarrow u$, which implies $T x_{n} \rightarrow T u=u$. Consequently, $T$ is continuous at $u$.

We give an example.

Example 2 Let $\mathrm{X}=\{0,2,4,8\}$ and $(\mathrm{X}, \mathcal{S})$ be the $\mathrm{S}$-metric space defined as in Example 1. Let us define the self-mapping $\mathrm{T}: \mathrm{X} \rightarrow \mathrm{X}$ as

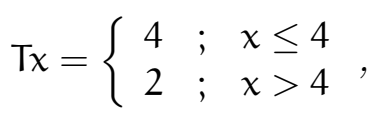

for all $\mathrm{x} \in\{0,2,4,8\}$. Then $\mathrm{T}$ satisfies the conditions of Theorem 1 with $\mathrm{a}=$ $\frac{3}{4}, \mathrm{~b}=\mathrm{c}=0$ and has a unique fixed point $\mathrm{x}=4$. Indeed, we get the following table:

$$
\begin{aligned}
& \mathcal{S}(\mathrm{T} x, \mathrm{~T} x, \mathrm{~T} y)=0 \quad \text { and } \quad 3 \leq \mathrm{M}_{z}^{\mathrm{S}}(\mathrm{x}, \mathrm{y}) \leq 6 \text { when } \mathrm{x}, \mathrm{y} \leq 4 \\
& \mathcal{S}(\mathrm{Tx}, \mathrm{Tx}, \mathrm{Ty})=4 \text { and } 6 \leq \mathrm{M}_{z}^{\mathrm{S}}(\mathrm{x}, \mathrm{y}) \leq 12 \text { when } \mathrm{x} \leq 4, \mathrm{y}>4 \text {. } \\
& \mathcal{S}(\mathrm{T} x, \mathrm{~T} x, \mathrm{~T} y)=4 \text { and } 6 \leq \mathrm{M}_{z}^{\mathrm{S}}(\mathrm{x}, \mathrm{y}) \leq 12 \text { when } \mathrm{x}>4, \mathrm{y} \leq 4
\end{aligned}
$$


Hence T satisfies the conditions of Theorem 1 with

$$
\phi(t)=\left\{\begin{array}{lll}
5 & ; & t \geq 6 \\
\frac{t}{2} ; & t<6
\end{array}\right.
$$

and

$$
\delta(\varepsilon)=\left\{\begin{array}{ccc}
6 & ; & \varepsilon \geq 3 \\
6-\varepsilon & ; & \varepsilon<3
\end{array} .\right.
$$

Now we give the following results as the consequences of Theorem 1.

Corollary 1 Let $(\mathrm{X}, \mathcal{S})$ be a complete $\mathrm{S}$-metric space and $\mathrm{T}$ a self-mapping on $\mathrm{X}$ satisfying the conditions

i) $\mathcal{S}(\mathrm{T} x, \mathrm{~T} x, \mathrm{Ty})<\mathrm{M}_{z}^{\mathrm{S}}(\mathrm{x}, \mathrm{y})$ for any $\mathrm{x}, \mathrm{y} \in \mathrm{X}$ with $\mathrm{M}_{z}^{\mathrm{S}}(\mathrm{x}, \mathrm{y})>0$,

ii) There exists a $\delta=\delta(\varepsilon)>0$ such that $\varepsilon<M_{z}^{S}(x, y)<\varepsilon+\delta$ implies $\mathcal{S}(\mathrm{T} x, \mathrm{~T} x, \mathrm{Ty}) \leq \varepsilon$ for a given $\varepsilon>0$.

Then $\mathrm{T}$ has a unique fixed point $\mathrm{u} \in \mathrm{X}$. Also, $\mathrm{T}$ is discontinuous at $\mathrm{u}$ if and only if $\lim _{x \rightarrow u} M_{z}^{S}(x, u) \neq 0$.

Corollary 2 Let $(\mathrm{X}, \mathcal{S})$ be a complete $\mathrm{S}$-metric space and $\mathrm{T}$ a self-mapping on $\mathrm{X}$ satisfying the conditions

i) There exists a function $\phi: \mathbb{R}^{+} \rightarrow \mathbb{R}^{+}$such that $\phi(\mathcal{S}(\mathrm{x}, \mathrm{x}, \mathrm{y}))<\mathcal{S}(\mathrm{x}, \mathrm{x}, \mathrm{y})$ and $\mathcal{S}(\mathrm{T} x, \mathrm{~T} x, \mathrm{~T} y) \leq \phi(\mathcal{S}(x, x, y))$,

ii) There exists a $\delta=\delta(\varepsilon)>0$ such that $\varepsilon<t<\varepsilon+\delta$ implies $\phi(t) \leq \varepsilon$ for any $\mathrm{t}>0$ and a given $\varepsilon>0$.

Then $\mathrm{T}$ has a unique fixed point $\mathrm{u} \in \mathrm{X}$.

The following theorem shows that the power contraction of the type $M_{z}^{S}(x, y)$ allows also the possibility of discontinuity at the fixed point.

Theorem 2 Let $(\mathrm{X}, \mathcal{S})$ be a complete $\mathrm{S}$-metric space and $\mathrm{T}$ a self-mapping on $\mathrm{X}$ satisfying the conditions

i) There exists a function $\phi: \mathbb{R}^{+} \rightarrow \mathbb{R}^{+}$such that $\phi(\mathrm{t})<\mathrm{t}$ for each $\mathrm{t}>0$ and

$$
\mathcal{S}\left(T^{m} x, T^{m} x, T^{m} y\right) \leq \phi\left(M_{z}^{S^{*}}(x, y)\right),
$$

where

$$
M_{z}^{S^{*}}(x, y)=\max \left\{\begin{array}{c}
a \mathcal{S}(x, x, y), \frac{b}{2}\left[\mathcal{S}\left(x, x, T^{m} x\right)+\mathcal{S}\left(y, y, T^{m} y\right)\right] \\
\frac{c}{2}\left[\mathcal{S}\left(x, x, T^{m} y\right)+\mathcal{S}\left(y, y, T^{m} x\right)\right]
\end{array}\right\}
$$

for all $\mathrm{x}, \mathrm{y} \in \mathrm{X}$, 
ii) There exists a $\delta=\delta(\varepsilon)>0$ such that $\varepsilon<M_{z}^{S^{*}}(x, y)<\varepsilon+\delta$ implies $\mathcal{S}\left(\mathrm{T}^{\mathrm{m}} \mathrm{x}, \mathrm{T}^{\mathrm{m}} \mathrm{x}, \mathrm{T}^{\mathrm{m}} \mathrm{y}\right) \leq \varepsilon$ for a given $\varepsilon>0$.

Then $\mathrm{T}$ has a unique fixed point $\mathrm{u} \in \mathrm{X}$. Also, $\mathrm{T}$ is discontinuous at $\mathrm{u}$ if and only if $\lim _{x \rightarrow \mathfrak{u}} M_{z}^{S^{*}}(x, \mathfrak{u}) \neq 0$.

Proof. By Theorem 1, the function $T^{m}$ has a unique fixed point $u$. Hence we have

$$
\mathrm{Tu}=\mathrm{T}^{\mathrm{m}} \mathrm{u}=\mathrm{T}^{\mathrm{m}} \mathrm{Tu}
$$

and so $\mathrm{Tu}$ is another fixed point of $\mathrm{T}^{\mathrm{m}}$. From the uniqueness of the fixed point, we obtain $\mathrm{Tu}=\mathfrak{u}$, that is, $\mathrm{T}$ has a unique fixed point $\mathfrak{u}$.

We note that if the $S$-metric $\mathcal{S}$ generates a metric d then we consider Theorem 1 on the corresponding metric space as follows:

Theorem 3 Let $(\mathrm{X}, \mathrm{d})$ be a complete metric space and $\mathrm{T}$ a self-mapping on $\mathrm{X}$ satisfying the conditions

i) There exists a function $\phi: \mathbb{R}^{+} \rightarrow \mathbb{R}^{+}$such that $\phi(\mathrm{t})<\mathrm{t}$ for each $\mathrm{t}>0$ and

$$
d(T x, T y) \leq \phi\left(M_{z}(x, y)\right)
$$

for all $\mathrm{x}, \mathrm{y} \in \mathrm{X}$,

ii) There exists a $\delta=\delta(\varepsilon)>0$ such that $\varepsilon<M_{z}(x, y)<\varepsilon+\delta$ implies $\mathrm{d}(\mathrm{T} x, \mathrm{Ty}) \leq \varepsilon$ for a given $\varepsilon>0$.

Then $\mathrm{T}$ has a unique fixed point $\mathrm{u} \in \mathrm{X}$. Also, $\mathrm{T}$ is discontinuous at $\mathrm{u}$ if and only if $\lim _{x \rightarrow \mathfrak{u}} M_{z}(x, \mathfrak{u}) \neq 0$.

Proof. By the similar arguments used in the proof of Theorem 1, the proof can be easily obtained.

\section{An application to the fixed-circle problem}

In this section, we investigate new solutions to the fixed-circle problem raised by Özgür and Taş in [19] related to the geometric properties of the set Fix(T) for a self mapping $\mathrm{T}$ on an $\mathrm{S}$-metric space $(X, \mathcal{S})$. Some fixed-circle or fixeddisc results, as the direct solutions of this problem, have been studied using various methods on a metric space or some generalized metric spaces (see $[15,16,20,21,22,23,26,27,30,31,32])$.

Now we recall the notions of a circle and a disc on an $S$-metric space as follows:

$$
C_{x_{0}, r}^{S}=\left\{x \in X: \mathcal{S}\left(x, x, x_{0}\right)=r\right\}
$$


and

$$
\mathrm{D}_{\mathrm{x}_{0}, \mathrm{r}}^{\mathrm{S}}=\left\{x \in X: \mathcal{S}\left(x, x, x_{0}\right) \leq \mathrm{r}\right\}
$$

where $r \in[0, \infty)[20,29]$.

If $T x=x$ for all $x \in C_{x_{0}, r}^{S}$ (resp. $x \in D_{x_{0}, r}^{S}$ ) then the circle $C_{x_{0}, r}^{S}$ (resp. the disc $D_{x_{0}, r}^{S}$ ) is called as the fixed circle (resp. fixed disc) of $T$ (for more details see $[15,20])$.

We begin with the following definition.

Definition 2 A self-mapping $\mathrm{T}$ is called an $\mathcal{S}$-Zamfirescu type $\mathrm{x}_{0}$-mapping if there exist $\mathrm{x}_{0} \in \mathrm{X}$ and $\mathrm{a}, \mathrm{b} \in[0,1)$ such that

$\mathcal{S}(\mathrm{T} x, \mathrm{~T} x, x)>0 \Longrightarrow \mathcal{S}(\mathrm{T} x, \mathrm{~T} x, x) \leq \max \left\{\begin{array}{c}\mathrm{a} \mathcal{S}\left(x, x, x_{0}\right), \\ \frac{\mathrm{b}}{2}\left[\mathcal{S}\left(\mathrm{T}_{0}, \mathrm{~T} x_{0}, \mathrm{x}\right)+\mathcal{S}\left(\mathrm{T} x, \mathrm{~T} x, x_{0}\right)\right]\end{array}\right\}$, for all $\mathrm{x} \in \mathrm{X}$.

We define the following number:

$$
\rho:=\inf \{\mathcal{S}(T x, T x, x): T x \neq x, x \in X\} .
$$

Now we prove that the set Fix $(\mathrm{T})$ contains a circle (resp. a disc) by means of the number $\rho$.

Theorem 4 If $\mathrm{T}$ is an $\mathcal{S}$-Zamfirescu type $\mathrm{x}_{0}$-mapping with $\mathrm{x}_{0} \in \mathrm{X}$ and the condition

$$
\mathcal{S}\left(\mathrm{T} x, \mathrm{~T} x, x_{0}\right) \leq \rho
$$

holds for each $\mathrm{x} \in \mathrm{C}_{\mathrm{x}_{0}, \rho}^{\mathrm{S}}$ then $\mathrm{C}_{\mathrm{x}_{0}, \rho}^{\mathrm{S}}$ is a fixed circle of $\mathrm{T}$, that is, $\mathrm{C}_{\mathrm{x}_{0}, \rho}^{\mathrm{S}} \subset \mathrm{Fix}(\mathrm{T})$.

Proof. At first, we show that $x_{0}$ is a fixed point of $\mathrm{T}$. On the contrary, let $\mathrm{T} \mathrm{x}_{0} \neq$ $x_{0}$. Then we have $\mathcal{S}\left(T x_{0}, T x_{0}, x_{0}\right)>0$. By the definition of an $\mathcal{S}$-Zamfirescu type $x_{0}$-mapping and the condition (S1), we obtain

$$
\begin{aligned}
\mathcal{S}\left(T x_{0}, T x_{0}, x_{0}\right) & \leq \max \left\{a \mathcal{S}\left(x_{0}, x_{0}, x_{0}\right), \frac{b}{2}\left[\mathcal{S}\left(T x_{0}, T x_{0}, x_{0}\right)+\mathcal{S}\left(T x_{0}, T x_{0}, x_{0}\right)\right]\right\} \\
& =b \mathcal{S}\left(T x_{0}, T x_{0}, x_{0}\right),
\end{aligned}
$$

a contradiction because of $b \in[0,1)$. This shows that $T x_{0}=x_{0}$.

We have two cases:

Case 1: If $\rho=0$, then we get $C_{\chi_{0}, \rho}^{S}=\left\{x_{0}\right\}$ and clearly this is a fixed circle of $\mathrm{T}$. 
Case 2: Let $\rho>0$ and $x \in C_{x_{0}, \rho}^{S}$ be any point such that $T x \neq x$. Then we have

$$
\mathcal{S}(\mathrm{T} x, \mathrm{~T} x, x)>0
$$

and using the hypothesis we obtain,

$$
\begin{aligned}
\mathcal{S}(\mathrm{T} x, \mathrm{~T} x, x) & \leq \max \left\{\mathrm{a} \mathcal{S}\left(x, x, x_{0}\right), \frac{\mathrm{b}}{2}\left[\mathcal{S}\left(\mathrm{T} x_{0}, \mathrm{~T} x_{0}, x\right)+\mathcal{S}\left(\mathrm{T} x, \mathrm{~T} x, x_{0}\right)\right]\right\} \\
& \leq \max \{\mathrm{a} \rho, \mathrm{b} \rho\}<\rho,
\end{aligned}
$$

which is a contradiction with the definition of $\rho$. Hence it should be $T x=\chi$ whence $C_{\chi_{0}, \rho}^{S}$ is a fixed circle of $T$.

Corollary 3 If $\mathrm{T}$ is an $\mathcal{S}$-Zamfirescu type $\mathrm{x}_{0}$-mapping with $\mathrm{x}_{0} \in \mathrm{X}$ and the condition

$$
\mathcal{S}\left(\mathrm{T} x, \mathrm{~T} x, x_{0}\right) \leq \rho
$$

holds for each $\mathrm{x} \in \mathrm{D}_{\mathrm{x}_{0}, \rho}^{S}$ then $\mathrm{D}_{\chi_{0}, \rho}^{S}$ is a fixed disc of $\mathrm{T}$, that is, $\mathrm{D}_{\mathrm{x}_{0}, \rho}^{S} \subset \operatorname{Fix}(\mathrm{T})$.

Now we give an illustrative example to show the effectiveness of our results.

Example 3 Let $\mathrm{X}=\mathbb{R}$ and $(\mathrm{X}, \mathcal{S})$ be the $\mathrm{S}$-metric space defined as in Example 1. Let us define the self-mapping $\mathrm{T}: \mathrm{X} \rightarrow \mathrm{X}$ as

$$
T x=\left\{\begin{array}{ccc}
x & ; & x \in[-3,3] \\
x+1 & ; & x \notin[-3,3]
\end{array},\right.
$$

for all $\mathrm{x} \in \mathbb{R}$. Then $\mathrm{T}$ is an $\mathcal{S}$-Zamfirescu type $\mathrm{x}_{0}$-mapping with $\mathrm{x}_{0}=0, \mathrm{a}=\frac{1}{2}$ and $\mathrm{b}=0$. Indeed, we get

$$
\mathcal{S}(\mathrm{T} x, \mathrm{~T} x, x)=2|\mathrm{~T} x-x|=2>0,
$$

for all $x \in(-\infty,-3) \cup(3, \infty)$. So we obtain

$$
\begin{aligned}
\mathcal{S}(\mathrm{T} x, \mathrm{~T} x, x) & =2 \leq \max \left\{\mathrm{aS}(x, x, 0), \frac{\mathrm{b}}{2}[\mathcal{S}(0,0, x)+\mathcal{S}(x+1, x+1,0)]\right\} \\
& =\frac{1}{2} \cdot 2|x| .
\end{aligned}
$$

Also we have

$$
\rho=\inf \{\mathcal{S}(T x, T x, x): T x \neq x, x \in X\}=2
$$

and

$$
\mathcal{S}(\mathrm{T} x, \mathrm{~T} x, 0)=\mathcal{S}(x, x, 0) \leq 2
$$

for all $x \in \mathrm{C}_{0,2}^{S}=\{x: \mathcal{S}(x, x, 0)=2\}=\{x: 2|x|=2\}=\{x:|x|=1\}$. Consequently, $\mathrm{T}$ fixes the circle $\mathrm{C}_{0,2}^{\mathrm{S}}$ and the disc $\mathrm{D}_{0,2}^{\mathrm{S}}$. 


\section{Acknowledgement}

This work is supported by the Scientific Research Projects Unit of Ballkesir University under the project numbers BAP 2018 /019 and BAP 2018 /021.

\section{References}

[1] R. K. Bisht and R. P. Pant, A remark on discontinuity at fixed point, $J$. Math. Anal. Appl., 445 (2017), 1239-1242.

[2] R. K. Bisht and R. P. Pant, Contractive definitions and discontinuity at fixed point, Appl. Gen. Topol., 18 (1) (2017), 173-182.

[3] R. K. Bisht and N. Hussain, A note on convex contraction mappings and discontinuity at fixed point, J. Math. Anal., 8 (4) (2017), 90-96.

[4] R. K. Bisht and V. Rakočević, Generalized Meir-Keeler type contractions and discontinuity at fixed point, Fixed Point Theory, 19 (1) (2018), 57-64.

[5] R. K. Bisht, $(\varepsilon-\delta)$ conditions and fixed point theorems, Tbil. Math. J. 12 (3) (2019), 39-49.

[6] R. K. Bisht and N. Özgür, Geometric properties of discontinuous fixed point set of $(\epsilon-\delta)$ contractions and applications to neural networks, Aequationes Math., 94 (5) (2020), 847-863. https://doi.org/10.1007/s00010019-00680-7

[7] M. Forti and P. Nistri, Global convergence of neural networks with discontinuous neuron activations, IEEE Trans. Circuits Syst. I, Fundam. Theory Appl., 50 (11) (2003), 1421-1435.

[8] A. Gupta, Cyclic contraction on S-metric space, Int. J. Anal. Appl., 3 (2) (2013), 119-130.

[9] N. T. Hieu, N. T. Ly and N. V. Dung, A generalization of Ciric quasicontractions for maps on S-metric spaces, Thai J. Math., 13 (2) (2015), 369-380.

[10] J. Jachymski, Common fixed point theorems for some families of maps, Indian J. Pure Appl. Math., 25 (1994), 925-937.

[11] J. Jachymski, Equivalent conditions and Meir-Keeler type theorems, $J$. Math. Anal. Appl., 194 (1995), 293-303. 
[12] X. Liu, T. Chen, J. Cao and W. Lu, Dissipativity and quasisynchronization for neural networks with discontinuous activations and parameter mismatches, Neural Networks, 24 (10) (2011), 1013-1021.

[13] W. Lu and T. Chen, Dynamical behaviors of Cohen-Grossberg neural networks with discontinuous activation functions, Neural Networks, 18 (3) (2005), 231-242.

[14] W. Lu and T. Chen, Dynamical behaviors of delayed neural network systems with discontinuous activation functions, Neural Computation, 18 (3) (2006), 683-708.

[15] N. Mlaiki, U. Çelik, N. Taş, N. Y. Özgür and A. Mukheimer, Wardowski type contractions and the fixed-circle problem on S-metric spaces, $J$. Math., 2018, Article ID 9127486.

[16] N. Mlaiki, N. Taş and N. Y. Özgür, On the fixed-circle problem and Khan type contractions, Axioms, 7 (2018), 80.

[17] N. Y. Özgür and N. Taş, Some new contractive mappings on S-metric spaces and their relationships with the mapping (S25), Math. Sci. (Springer), 11 (1) (2017), 7-16.

[18] N. Y. Özgür, N. Taş, Generalizations of metric spaces: from the fixedpoint theory to the fixed-circle theory, In: Rassias T. (eds) Applications of Nonlinear Analysis. Springer Optimization and Its Applications, vol 134. Springer, Cham, 2018.

[19] N. Y. Özgür and N. Taş, Some fixed-circle theorems on metric spaces, Bull. Malays. Math. Sci. Soc., 42 (4) (2019), 1433-1449.

[20] N. Y. Özgür and N. Taş, Fixed-circle problem on S-metric spaces with a geometric viewpoint, Facta Universitatis. Series: Mathematics and Informatics, 34 (3) (2019), 459-472.

[21] N. Y. Özgür, N. Taş and U. Çelik, New fixed-circle results on S-metric spaces, Bull. Math. Anal. Appl., 9 (2) (2017), 10-23.

[22] N. Y. Özgür and N. Taş, Some fixed-circle theorems and discontinuity at fixed circle, AIP Conference Proceedings, 1926, 020048 (2018).

[23] N. Y. Özgür, Fixed-disc results via simulation functions, Turkish J. Math., 43 (6) (2019), 2794-2805. 
[24] A. Pant and R. P. Pant, Fixed points and continuity of contractive maps, Filomat, 31 (11) (2017), 3501-3506.

[25] R. P. Pant, Discontinuity and fixed points, J. Math. Anal. Appl., 240 (1999), 284-289.

[26] R. P. Pant, N.Y. Özgür and N. Taş, On discontinuity problem at fixed point, Bull. Malays. Math. Sci. Soc., 43 (1) (2020), 499-517.

[27] R. P. Pant, N. Y. Özgür and N. Taş, Discontinuity at fixed points with applications, Bulletin of the Belgian Mathematical Society-Simon Stevin, 26 (4) (2019), 571-589.

[28] B. E. Rhoades, Contractive definitions and continuity, Fixed point theory and its applications (Berkeley, CA, 1986), 233-245, Contemp. Math., 72, Amer. Math. Soc., Providence, RI, 1988.

[29] S. Sedghi, N. Shobe and A. Aliouche, A generalization of fixed point theorems in S-metric spaces, Mat. Vesnik, 64 (3) (2012), 258-266.

[30] N. Taş, N. Y. Özgür and N. Mlaiki, New types of $F_{C}$-contractions and the fixed-circle problem, Mathematics, 6 (10) (2018), 188.

[31] N. Taş, Suzuki-Berinde type fixed-point and fixed-circle results on Smetric spaces, J. Linear Topol. Algebra, 7 (3) (2018), 233-244.

[32] N. Taş, Various types of fixed-point theorems on S-metric spaces, $J$. BAUN Inst. Sci. Technol., 20 (2) (2018), 211-223.

[33] T. Zamfirescu, Fix point theorems in metric spaces, Arch. Math., 23 (1972), 292-298. 